\title{
Einschränkung des Versicherungsumfangs in der deutschen gesetzlichen Unfallversicherung im Hinblick auf Wegeunfälle und Berufskrankheiten
}

\author{
Prof. Dr. Maximilian Fuchs
}

\section{Vorbemerkung}

Wegeunfälle und Berufskrankheiten stellen dar, was man im Versicherungswesen als Versicherungsfälle bezeichnet. Der Versicherungsfall ist die zentrale Kategorie jeder Versicherung ${ }^{1}$. Versicherung hat es mit der Bewältigung von Risiken und ihrer Verwirklichung zu tun. Der Versicherungsfall ist die Verwirklichung des versicherten Risikos. Und das Vorliegen des Versicherungsfalls löst die Leistungen aus. Vor diesem Hintergrund leuchtet es ein, dass die rechtliche Fixierung des Versicherungsfalls von elementarer Bedeutung für die Funktionsweise des betreffenden Versicherungszweiges ist.

In diesem Sinne möchte ich auch mein Referat angelegt wissen. Im Vordergrund steht die positiv-rechtliche Darstellung der beiden Versicherungsfälle. Ebenso notwendig ist aber gleichzeitig die Frage nach der Rationalität der zu besprechenden Regelungen. Diese Rationalität kann nur bewertet werden, wenn wir die beiden Versicherungsfälle im Rahmen des gesamten Systems betrachten, das die gesetzliche Unfallversicherung bildet. Die Bewältigung von Körperschäden ${ }^{2}$ wird ja auch von anderen Zweigen der Sozialversicherung geleistet, etwa der Krankenversicherung und Rentenversicherung. Was die Unfallversicherung zu einem speziellen Entschädigungssystem macht, liegt in der Besonderheit begründet ${ }^{3}$, dass

- $\quad$ aus dem großen Feld eintretender Körperschäden diejenigen herausgefiltert werden, die sich kausal auf eine versicherte Tätigkeit zurückführen lassen und

- die Verantwortung für die Kompensation dieser Schäden ausschließlich den Unternehmen überantwortet ist.

Nach der Konzeption des Gesetzes besteht also durchaus eine Verantwortlichkeit der Unternehmen im Sinne der bürgerlichen Haftungsregelungen. Diese Haftungsverant-

1 Zur zentralen Rolle des Versicherungsfalls Weyers, Versicherungsvertragsrecht, 2. Aufl. 1995 Rn. 34 und 551.

2 Zum System der Bewältigung von Körperschäden im deutschen Recht s. Fuchs, Zivilrecht und Sozialrecht, 1992, S. $168 \mathrm{ff}$.

$3 \mathrm{Zu}$ den Grundprinzipien und -mechanismen der gesetzlichen Unfallversicherung s. Gitter, Schadensausgleich im Arbeitsunfallrecht, 1969; Fuchs, Die Legitimation der gesetzlichen Unfallversicherung, SGB 1995, 421 ff.; ders., Struktur und Legitimation der gesetzlichen Unfallversicherung: Eine rechtsvergleichende Betrachtung, in: Internationale Revue für Soziale Sicherheit, 1997, 19 ff. 
wortung wird aber im Regelfall von der Unfallversicherung abgelöst, weshalb sie auch allein der Unternehmer durch Beiträge zu finanzieren hat. Das ist das, was man als Haftungsablösung durch Versicherungsschutz ${ }^{4}$ bezeichnet hat. In dieses versicherungsrechtliche Schema müssen sich auch die Versicherungsfälle des Wegeunfalls und der Berufskrankheiten einfügen.

\section{Der Wegeunfall}

\section{Tatbestand}

Die Grundnorm des Wegeunfalls in $\S 8$ Abs. 2 Nr. 1 SGB VII sagt, dass versicherte Tätigkeit auch das Zurücklegen des mit der versicherten Tätigkeit zusammenhängenden unmittelbaren Weges nach und von dem Ort der Tätigkeit ist ${ }^{5}$. Eingeweihte wissen, dass diese Vorschrift viele Auslegungsprobleme aufgeworfen hat, die nicht zuletzt damit zusammenhängen, dass die Vorschrift nur einen Punkt der Wegstrecke, nämlich den Ort der Tätigkeit, benennt, den anderen Punkt offen lässt. Deshalb ist in der Rechtsprechung eine reiche Kasuistik entstanden ${ }^{6}$. Wenn man versucht, eine Struktur des bislang judizierten zu erreichen, kann man diese vielleicht wie folgt zusammenfassen:

Grundsätzlich ist die Wegstrecke markiert als der direkte Weg zwischen Wohnung und Ort der Tätigkeit und zurück. Ausgangspunkt kann aber unter bestimmten Voraussetzungen auch ein so genannter dritter $\mathrm{Ort}^{7}$ sein. Dann muss allerdings ein innerer $\mathrm{Zu}$ sammenhang mit der Tätigkeit gegeben sein.

Sodann werden im Zusammenhang mit der Bestimmung des versicherten Weges zwei Begriffe verwandt, der so genannte Umweg und der so genannte Abweg8 ${ }^{8}$. Beim Umweg sind Ausgangspunkt und Endpunkt der Wegstrecke identisch mit der üblichen, aber verlassen wird die direkte Route. Deshalb ist der Weg länger. Hier verlangt die Rechtsprechung (BSG NZS 2002, 161) eine wertende Betrachtung, ob hier noch eine vernünftige Relation zwischen Umweg und direktem Weg besteht und ob nach der allgemeinen Verkehrsanschauung dieser Weg noch als versicherter Weg gelten kann.

4 Ausführlich dazu Gitter/Nunius in: Schulin (Hg.), Handbuch des Sozialversicherungsrechts, Bd. 2 Unfallversicherungsrecht, 1996, § 5 Rn. 51-58.

$5 \mathrm{Zu}$ einer knappen Zusammenfassung und Darstellung der rechtlichen Struktur des Wegeunfalls s. Schmitt, Unfallversicherung in: von Maydell/Ruland (Hg.), Sozialrechtshandbuch, 3. Aufl. 2003, C $15 \mathrm{Rn} .125 \mathrm{ff}$.

6 Übersicht über die Rechtsprechung bei Bereiter-Hahn/Mehrtens, Gesetzliche Unfallversicherung, $\S 8 \mathrm{Rn} .12 \mathrm{ff}$.

7 Zu Konzeption und Anwendungsbeispielen des „Dritten Ortes“ Franke/Molkentin (Hg.), Sozialgesetzbuch VII, 2. Aufl., 2007, § 8 Rn. 164 ff.

$8 \mathrm{Zu}$ diesen Kategorien Muckel, Sozialrecht, 2. Aufl., 2007, § 10 Rn. 58. 
Beim Abweg 9 besteht Deckungsgleichheit mit dem unmittelbaren Weg nur im Hinblick auf den Ausgangspunkt, sein Ziel liegt in anderer Richtung. Nach Erreichen des Ziels führt allerdings der Weg zum Ausgangspunkt zurück. Der Abweg ist grundsätzlich unversichert, führt er allerdings irgendwann wieder auf den direkten Weg zurück, kann an dieser Stelle erneut der Unfallversicherungsschutz einsetzen.

Ein letztes Problemfeld wird gerne unter dem Stichwort der Unterbrechung des versicherten Weges ${ }^{10}$ zusammengefasst. Darunter versteht man die auf der direkten Wegstrecke oder aber auch bei Zurücklegen eines Umweges oder Abweges vorgenommene private, eigenwirtschaftliche Tätigkeit des Versicherten. Diese eigenwirtschaftliche Tätigkeit, soweit sie nicht bloß ganz geringfügiger Natur ist, ist natürlich nicht versichert. Die Rückkehr auf den direkten Weg lässt aber den Versicherungsschutz wieder aufleben. Dies gilt allerdings nur dann, wenn die Unterbrechung nicht zu einer so genannten Lösung (Loslösung) von der versicherten Tätigkeit geführt hat. Bei der Beurteilung dieser Frage sollen verschiedene Momente eine Rolle spielen, ganz besonders aber das Zeitelement. Bei zwei Stunden beginnt jedenfalls die sehr kritische Grenze ${ }^{11}$.

Um die positiv-rechtliche Darstellung zu komplettieren, sei darauf hingewiesen, dass $\S 8$ Abs. 2 Nr. 1-5 SGB VII noch weitere Wege als versicherte Tätigkeiten betrachtet ${ }^{12}$. Man könnte sagen, es sind bestimmte Umwege von Gesetzes wegen zu versicherten Wegen gemacht. Die Gründe hierfür liegen einmal darin, dass der Umweg wegen der Betreuung von Kindern durch dritte Personen notwendig ist oder auf eine Fahrgemeinschaft zurückzuführen ist oder weil ständige Familienwohnung und Wohnung am Ort der Tätigkeit des Versicherten auseinander fallen oder weil Wege zurückzulegen sind, um Arbeitsgerät oder Schutzausrüstungen anzuschaffen oder erneuern zu lassen.

\section{Systemgerechtigkeit}

Vor diesem rechtlichen Hintergrund möchte ich nun einige Bemerkungen anschließen, die die Systemgerechtigkeit des Wegeunfalls betreffen. Gitter hat in seinem Werk über Schadensausgleich im Arbeitsunfallrecht, erschienen 1969, geschrieben, dass selbst in europäischen Unfallversicherungsgesetzen, die den Schutzgedanken umfassender verwirklichen, als es das deutsche Recht tut, der Unfall auf dem Wege zwischen Wohnung und Arbeitsstätte vom Versicherungsschutz ausgeklammert wird, weil es sich um ein allgemeines Lebensrisiko handle ${ }^{13}$. Oder aber der Wegeunfall ist in die Unfallversicherung einbezogen, muss aber - weil Risiko des Arbeitnehmers - auch von die-

$9 \mathrm{Zu}$ den Fallkonstellationen Franke/Molkentin (Hg.) (Fn. 7), § 8 Rn. 193 ff.

$10 \mathrm{Zu}$ den Rechtsfolgen bei Unterbrechung des Weges s. Plagemann/Radtke-Schwenzer, Gesetzliche Unfallversicherung, 2. Aufl. 2007, 2. Kap. Rn. 53.

11 Vgl. BSG SozR $3-2700 \S 8$ Nr. 14.

12 Vgl. dazu Franke/Molkentin (Hg.) (Fn. 7), Rn. 205 ff.

13 Gitter (Fn. 3), S. 86. 
sem finanziert werden. In Deutschland - so Gitter weiter - wird für den Versicherungsschutz bei Wegeunfällen vorgebracht, dass das Risiko des Arbeitsweges angesichts der technisch und soziologisch bedingten heutigen Verteilung von Wohnbezirken und Geschäfts- und Industriezentren typischerweise dem Berufsrisiko näher stehe als der privaten Sphäre ${ }^{14}$. Gitter selbst möchte im Prinzip die Regelung über den Wegeunfall aufrechterhalten, de lege ferenda aber eine gesetzliche Kürzungsmöglichkeit im Falle groben Verschuldens oder Trunkenheit bei Wegeunfällen auch für das deutsche Recht vorsehen ${ }^{15}$.

Bis in die jüngste Vergangenheit ist in Deutschland verständlicherweise die jetzige Regelung von Arbeitgeberseite kritisiert worden ${ }^{16}$. In der Tat muss man zugestehen, dass die jetzt vorgenommene Risikoverteilung, die auf eine Bejahung der Risikoverantwortung von Arbeitgebern hinausläuft, nur schwer zu begründen ist. Umso mehr, wenn man insbesondere an die gesetzlichen Ausweitungen versicherter Umwege, die etwa durch Kinderbetreuung veranlasst sind, denkt. Würde man die Wegeunfälle aus der Unfallversicherung herausnehmen, würden die Kosten dennoch anfallen. Sie wären von der gesetzlichen Krankenversicherung und Rentenversicherung zu tragen. An diesen Kosten wären Arbeitnehmer und Arbeitgeber über Beiträge in gleichem Umfang beteiligt.

\section{Die Berufskrankheit}

\section{Der historische Hintergrund}

Ich habe mich bei der Darstellung der Berufskrankheitenentschädigung für einen wenn der Ausdruck nicht zu hoch gegriffen ist - historischen Ansatz entschieden. Dies ist keine willkürliche Entscheidung für eine bestimmte Methode der Betrachtung. Das Berufskrankheitenrecht ist vielmehr ein besonders augenfälliges Lehrstück von Sozialpolitik und ihr folgend Formung von Sozialrecht. Und deshalb verschafft uns die Kenntnis der Geschichte des Berufskrankheitenrechts ${ }^{17}$ nahezu das gesamte Wissen, um das aktuell geltende Berufskrankheitenrecht einschließlich der Reformvorschläge zu verstehen.

Deshalb begebe ich mich für einen Augenblick zurück in die Anfänge der Unfallversicherung und der Herausbildung von Regelungen zur Berufskrankheitenentschädigung. Wie Sie wissen beinhaltete das Unfallversicherungsgesetz von 1884 keine Berufskrank-

14 Gitter (Fn. 3), S. 87 f.

$15 \operatorname{Gitter}$ (Fn. 3), S. 88 f.

16 Vgl. zu dieser Kritik und zu einer Antwort hierauf unter umfassender Einbeziehung von Geschichte der Regelung und dogmatischer Handhabung Kranig/Aulmann, Das Wegeunfallrisiko als Gegenstand der gesetzlichen Unfallversicherung, Teil 1 und 2, NZS 1995, 203 ff. und 255 ff.

17 Vgl. dazu die Beiträge Milles/Müller (Hg.), in: Berufsarbeit und Krankheit, 1985. 
heitenentschädigung. Das Problem war gleichwohl bei den Beratungen zum Gesetz bekannt und Forderungen, insbesondere aus den Reihen der Gewerkschaften sind erhoben worden ${ }^{18}$. Nicht zuletzt hatte auch die Ärzteschaft, die insbesondere in den Fabriken der chemischen Industrie tätig war, die Notwendigkeit betont. In den Jahren nach der Verabschiedung des Gesetzes hat sich die Diskussion intensiviert. Der Begriff der Gewerbekrankheiten fand Eingang in die wissenschaftliche und später auch politische Diskussion ${ }^{19}$. Der Begriff spiegelte das Bewusstsein wider, dass vor allem der Umgang mit bestimmten Stoffen wie Blei, Phosphor, Quecksilber und natürlich Staub in den Bergwerken und Steinbrüchen die Ursache für das Auftreten bestimmter Krankheiten darstellte. Für die rechtliche Bewältigung der damit verbundenen Phänomene in der Zeit nach der Verabschiedung des UVG ist die Rechtsprechung des für so genannte Rekurse zuständigen Reichsversicherungsamtes aufschlussreich. Dieser Rechtsprechung liegt eine klare Scheidung von Arbeitsunfällen und Gewerbekrankheiten zugrunde. Schon damals wurde die Definition herausgearbeitet, die bis heute Gültigkeit besitzt. Arbeitsunfall ist ein plötzliches von außen auf den Körper einwirkendes Ereignis ${ }^{20}$. Diese Sichtweise spiegelt sich in der Struktur der Entscheidungen wider. Liegen diese definitorischen Voraussetzungen nicht vor, kann es sich nur um eine Gewerbekrankheit handeln, die nach dem Gesetz nicht entschädigungsfähig ist. Die Rechtsprechung ist insofern nicht sehr konsequent, als vereinzelt doch auch Entscheidungen gefällt wurden, bei denen der Unfallbegriff extensiv ausgelegt wurde, um eine Entschädigung zu ermöglichen, obwohl streng genommen eine Gewerbekrankheit vorgelegen hat ${ }^{21}$. Sicherlich dürften für solche Urteile auch Auswirkungen der z.T. heftig und hitzig geführten Debatte um die Notwendigkeit einer Berufskrankheitenentschädigung eine Rolle gespielt haben. Unbehagen und Kritik an der Rechtsprechung des Reichsversicherungsamtes, aber auch die immer zahlreicher werdenden Veröffentlichungen von Medizinern führen schließlich dazu, dass die Berufskrankheitenfrage zu einem Gegenstand der Beratungen im Reichstag wird ${ }^{22}$. Mit dem Hinweis auf die Rolle der Medizin weise ich auf ein weiteres Element, das auch im aktuellen Recht der Berufskrankheitenentschädigung gegenwärtig ist. Wie wir sehen werden, sind die Erkenntnisse der medizinischen Wissenschaft sogar zu einem Tatbestandsmerkmal der gegenwärtigen Anspruchsnorm geworden. Das weist auf den später noch zu unterstreichenden Aspekt hin, dass die Entschädigung von Berufskrankheiten nicht, wie etwa 1884 von den Befürwortern gefordert, eine Billigkeitsentschädigung darstellt, sondern eine Reaktion auf objektiv konstatierbare Phänomene bei der Verrichtung abhängiger Arbeit.

18 Vgl. Hohmann, Berufskrankheiten im Prozeß der Modernisierung, 1997, S. 50 ff.

19 Vgl. zum Folgenden Hohmann (Fn. 18), S. 74 ff.

20 Vgl. RVA in EuM Bd. 2, S. 188. Diese Auffassung vertrat auch die damalige Lehre, vgl. Rosin, Der Begriff des Betriebsunfalls als Grundlage des Entschädigungsanspruchs nach den Reichsgesetzen über die Unfallversicherung, in AÖR Bd. III S. 291 ff.; Piloty, Das Reichsunfallversicherungsrecht, dessen Entstehungsgeschichte und System, Bd. 1, 1890, S. 180.

21 S. dazu Hohmann (Fn. 18), S. 25 ff.

22 Hohmann (Fn. 18), S. 76 ff. 
Die Reichstagsdiskussion nimmt mit Beginn des 20. Jahrhunderts an Intensität zu. Es dauert aber ein ganzes Jahrzehnt, bis eine Mehrheit für eine Berufskrankheitenentschädigung gefunden werden kann. Zunächst sind es zwei Hauptargumente, die einer rascheren Lösung im Wege stehen. Zum einen ist es das auch im Übrigen für die Sozialversicherung immer wieder herangezogene Argument der allzu starken Belastung der Unternehmen ${ }^{23}$ zum andern die ebenfalls die gesamte Geschichte des Berufskrankheitenrechts bis zum heutigen Tag prägende Schwierigkeit, festzulegen, ob eine Erkrankung berufsbedingt ist oder nicht. Die im Jahre 1911 verabschiedete Reichsversicherungsordnung 24 enthielt in $\S 547$ die Ermächtigung, wonach durch Beschluss des Bundesrats die Unfallversicherung auf bestimmte gewerbliche Berufskrankheiten ausgedehnt werden kann. Der Bundesrat soll hierzu besondere Vorschriften erlassen. Damit wurde der Grundstein für das bis auf den heutigen Tag geltende so genannte Listenoder Enumerationsprinzip gelegt. Entschädigt werden können danach nur solche Krankheiten, die in die Liste aufgenommen sind. Verschiedene Schwierigkeiten, ganz besonders natürlich der Erste Weltkrieg, verhinderten, dass von dieser Ermächtigung rasch Gebrauch gemacht wurde. Und so dauerte es bis zum Jahre 1925, dass die erste Verordnung über die Ausdehnung der Unfallversicherung auf gewerbliche Berufskrankheiten erlassen wurde. Die entscheidenden Vorschriften der $\S \S 3$ und 4 dieser VO lauteten:

„Der Versicherung gegen eine gewerbliche Berufskrankheit unterliegen nur die neben der Krank-

heit in Spalte III der Anlage I aufgeführten Betriebe, sofern sie unter die Gewerbe-

Unfallversicherung fallen.

Eine Entschädigung wird gewährt, wenn die Krankheit durch berufliche Beschäftigung in einem der Versicherung gegen die Krankheit unterliegenden Betriebe verursacht ist".

Verglichen mit der Regelung des $§ 9$ SGB VII, die wir uns später anzusehen haben, kann man feststellen, dass alle wesentlichen Elemente der Berufskrankheitenentschädigung im Sinne einer Listenregelung und der maßgeblichen Kriterien für ihre Aufnahme bereits in diesen Vorschriften enthalten sind. In diesem Zusammenhang erwähne ich noch eine weitere Bestimmung, die eine Thematik beinhaltet, die uns später noch intensiver beschäftigen wird. $\S 13$ der VO beinhaltete eine begrenzte Rückwirkung der Verordnung und damit Antwort auf eines der nach wie vor umstrittensten Probleme der Berufskrankheitenentschädigung. Diese Rückwirkung bezog sich aber nur auf einen sehr bescheidenen Zeitraum, so dass bald erhebliche Kritik an dem Rechtszustand geäuBert wurde, was zu einer Änderung der VO im Jahre 1929 führte, mit dem Ergebnis, dass der Stichtag auf den 31. Dezember 1919 zurückverlegt wurde.

23 Vgl. dazu Fuchs (Fn. 2), S. 28.

24 Vgl. zur Kodifikation der Sozialversicherung durch die Reichsversicherungsordnung Fuchs, Die Reichsversicherungsordnung als sozialrechtliche Kodifikation, in: FS für Landau, 2000, S. 883 ff. 


\section{Der Tatbestand des $\S 9$ Abs. 1 SGB VII}

\section{a) Die Struktur der Berufskrankheit}

Ich denke, dass mit diesem - notgedrungen kursorischen - Exkurs schon das gesamte Panorama der Probleme auch des aktuellen Berufskrankheitenrechts, wie wir sie im SGB VII finden, abgesteckt wurde.

Wenn wir jetzt den Blick auf § 9 SGB VII richten, stellen wir fest, dass wir uns nach wie vor auf Schienen bewegen, die 1911 bzw. 1925 gelegt wurden, allerdings haben die Schienen nicht unbedeutende Stärkungen erfahren. Naturgemäß ist das Regelungsanliegen gleich geblieben, das darin besteht, den Krankheiten, die aus dem Zuständigkeitsbereich der Krankenversicherung herausfallen sollen, Namen und Struktur zu geben. Dies tut $\S 9$ Abs. 1 S. 1 SGB VII ganz in der Tradition seines Vorläufers, indem Krankheiten als Berufskrankheiten in einer Rechtsverordnung aufgelistet sein müssen und diese sich auf eine versicherte Tätigkeit zurückführen lassen ${ }^{25}$.

Was allerdings die Kriterien der Aufnahme einer Krankheit in die Verordnung anbelangt, haben wir uns gegenüber dem früheren Recht für eine stärkere Differenzierung entschieden. Wenn wir $\S 9$ Abs. 1 S. 2 SGB VII betrachten, stellen wir fest, dass der Gesetzgeber das Abgrenzungsproblem zwischen Krankheiten, die ganz allgemein die Bevölkerung treffen können, und solchen, die in spezifischer Weise den versicherten Personenkreis treffen, stärker in den Mittelpunkt gerückt hat. Mit dem begrifflichen Verlangen nach einer erheblich höheren Intensität der Einwirkung führt der Gesetzgeber dem Verordnungsgeber schon in der tatbestandlichen Formulierung das Problem der Risikozuordnung vor Augen ${ }^{26}$. Um dieses Ziel zu erreichen, enthält das Gesetz sozusagen Filter, mit deren Hilfe es möglich sein soll, jeden treffende Erkrankungen aus der Entschädigung herauszuhalten. Dies geschieht einmal dadurch, dass Berufskrankheiten nur durch bestimmte Einwirkungen von außen indiziert sein müssen. Die BKVO unterscheidet hierzu durch chemische Einwirkungen, mechanische Einwirkungen, durch Infektionserreger, durch anorganische Stäube und durch allergisierende Stoffe entstandene Erkrankungen. Bei Erkrankungen, die auch sonst in der Bevölkerung häufig auftreten, muss tatbestandlich die besondere Exposition normiert werden. Typisch hierfür ist etwa der Bereich der Meniskusschäden, die bekanntlich viele Menschen treffen und etwa auf starke sportliche Beanspruchung zurückzuführen sein können. Deshalb verlangt der einschlägige Tatbestand für die Anerkennung von Meniskusschäden als Berufskrankheit das Vorhandensein mehrjähriger andauernder oder häufig wiederkehrender, die Kniegelenke überdurchschnittlich belastender Tätigkeiten ${ }^{27}$. Ich sollte hinzusetzen, dass es einfach ist, das Abgrenzungsproblem zu formulieren, viel schwieriger aber, eine für die

25 Vgl. dazu Kater/Leube, Gesetzliche Unfallversicherung SGB VII § 9 Rn 2 ff.

26 Eingehend zur praktischen Handhabung dieses Kriteriums BSG ZfS 2002, 334.

27 Vgl. Nr. 2102 BKVO. 
Praxis taugliche Quantifizierung vorzunehmen. Deshalb sind in der Vergangenheit so genannte Dosiskonzepte 28 entwickelt worden, die z.T. bereits Eingang in die BKVO gefunden haben. So wird Lungenkrebs oder Kehlkopfkrebs nur dann als Berufskrankheit anerkannt, wenn der Nachweis der Einwirkung einer kumulativen Asbestfaserstaubdosis am Arbeitsplatz von mindestens 25 Faserjahren erbracht ist ${ }^{29}$.

Ein weiterer vom Gesetz vorgezeichneter Filter ist die Begrenzung von Berufskrankheiten auf Tätigkeiten in bestimmten Gefährdungsbereichen. Diese Idee ist nicht neu, sie war in der BKVO von 1925 verwirklicht, dort aber auf die Benennung bestimmter gefährdender Betriebe beschränkt. Demgegenüber wird heute auf bestimmte Gefährdungsbereiche abgestellt. Typisches Beispiel ist etwa das Auftreten von Infektionskrankheiten, die jedermann treffen können, zur Berufskrankheit aber dann werden, wenn der Versicherte etwa im Gesundheitsdienst, in der Wohlfahrtspflege oder in einem Laboratorium tätig war ${ }^{30}$.

Eine weitere Methode, um Berufskrankheiten von nicht dem Schutz der Unfallversicherung zu unterstellenden Allgemeinerkrankungen abzugrenzen, ist das Kriterium der Unterlassung aller Tätigkeiten, die für die Entstehung, die Verschlimmerung oder das Wiederaufleben der Krankheit ursächlich waren oder sein können. Dieses Kriterium, das ansatzweise schon in der dritten BKVO von 1936 eingeführt wurde, hat - und ich folge hier der Formulierung einer Entscheidung des BSG - die Funktion eines typisierten Kausalitätsanzeichens, aufgrund dessen die nicht für entschädigungswürdig gehaltenen leichten Fälle dieser Krankheit, die häufig nicht ihre Ursache in der Versichertentätigkeit finden, abgegrenzt werden sollen ${ }^{31}$. Der weitere Zweck derartiger Tatbestandsmerkmale ist präventiv. Damit soll eine Verschlimmerung der Krankheit mit der Folge einer erhöhten Entschädigungspflicht verhindert werden. Um auch hier ein Beispiel zu geben: Nr. 4301 der BKVO behandelt durch allergisierende Stoffe verursachte obstruktive Atemwegserkrankungen, die zur Unterlassung aller Tätigkeiten gezwungen haben, die für die Entstehung, die Verschlimmerung oder das Wiederaufleben der Krankheit ursächlich waren oder sein können. Im Zusammenhang mit den Tatbeständen, die eine Unterlassung der Tätigkeiten verlangen, ist auf die Bestimmung des § 9 Abs. 4 SGB VII aufmerksam zu machen. Um zu vermeiden, dass der Versicherte die Tätigkeit in Erwartung der Anerkennung als Berufskrankheit aufgibt, sich diese Erwartung aber nicht bestätigt, verpflichtet Abs. 4 den Unfallversicherungsträger vor Unterlassung einer noch verrichteten gefährdeten Tätigkeit darüber zu entscheiden, ob die übrigen Voraussetzungen der Anerkennung einer Berufskrankheit erfüllt sind.

Stets hängt die Aufnahme einer Berufskrankheit von den Erkenntnissen der medizinischen Wissenschaft ab. Eingangs wurde bereits darauf hingewiesen, dass der Gesetz-

28 Vgl. dazu Brackmann/Krasney, SGB VII, § 9 Rn 60 mit weiteren Literaturnachweisen.

29 Nr. 4104 BKVO.

30 Nr. 3101 ff. BKVO.

31 BSG SozR $2200 \S 551$ Nr. 10. Zum gesamten Fragenkomplex s. Becker, Der Unterlassungszwang bei Berufskrankheiten, 2003. 
geber keine sozusagen rein sozialpolitische Entscheidung bei der Normierung von Berufskrankheiten treffen sollte. Vielmehr sollten es die jeweiligen Fachwissenschaften der Medizin sein, die anhand der gesetzlichen Kriterien den Charakter einer Krankheit als Berufskrankheit zum Ausdruck bringen sollten.

\section{b) Kausalität}

Schon die gesetzliche Formulierung lässt erkennen, dass der Anspruch auf Entschädigung wegen Berufskrankheit einen Ursachenzusammenhang zwischen versicherter Tätigkeit und Einwirkungen einerseits und zwischen Einwirkungen und dem Auslösen einer Berufskrankheit andererseits voraussetzt. Im ersteren Falle sprechen wir von haftungsbegründender, in der zweiten Beziehung von haftungsausfüllender Kausalität. Damit befinden wir uns an einer wohlbekannten Stelle der Anspruchsprüfung im Unfallversicherungsrecht ${ }^{32}$. Das Unfallversicherungssystem wird ja deshalb bewusst als ein kausales System, im Gegensatz zu finalen Systemen, bezeichnet. Mit der Kausalitätsprüfung wird die endgültige Entscheidung über eine korrekte Risikozuordnung zum Unternehmen oder aber zum Einzelnen vorgenommen.

Begegnen uns schon beim Arbeitsunfall schwierige Kausalitätsprobleme, so nehmen diese Probleme vielleicht sogar noch stärker zu, wenn es um die Kausalbeziehungen bei Berufskrankheiten geht ${ }^{33}$. Dies hängt damit zusammen, dass die Krankheit sich ja gerade durch das nicht Punktuelle, das in der zeitlichen Dimension sich Abspielende auszeichnet. Die Frage, welche Voraussetzungen zur Annahme eines ursächlichen Zusammenhangs zwischen der schädigenden Einwirkung und der Erkrankung einer Berufskrankheit vorliegen müssen, ist unter Zuhilfenahme medizinischer, naturwissenschaftlicher und technischer Sachkunde nach dem im Entscheidungszeitpunkt aktuellen wissenschaftlichen Erkenntnisstand zu beantworten ${ }^{34}$. Als aktueller Erkenntnisstand sind solche durch Forschung und praktische Erfahrung gewonnenen Erkenntnisse anzusehen, die von der großen Mehrheit der auf dem betreffenden Gebiet tätigen Fachwissenschaftler anerkannt werden, über die also, von vereinzelten, nicht ins Gewicht fallenden Gegenstimmen abgesehen, Konsens besteht ${ }^{35}$.

Was die Beweisführung und -last anbelangt, gelten die gleichen Grundsätze wie beim Arbeitsunfall ${ }^{36}$. Das Bundessozialgericht hat es abgelehnt, für das Berufskrankheitenrecht spezielle Beweisregeln zu entwickeln ${ }^{37}$. Das bedeutet, dass für alle tat-

32 Umfassend zur Kausalitätslehre Schöpf, Multikausale Schäden in der gesetzlichen Unfallversicherung, 1995, S. 26 ff.

33 Vgl. dazu ausführlich Rothmann, Zurechnungsproblem im Berufskrankheitenrecht, 1995, S. 63 ff.

34 BSG SozR $3850 \S 51$ Nr. 9; Krasney/Udsching, Handbuch des sozialgerichtlichen Verfahrens, 4. Aufl. 2005, Kap. III Rn 47, 57.

35 BSG NZS 2007, 325, 327.

36 Ausführlich dazu Brackmann/Becker, SGB VII, § 9 Rn 202 ff.

37 BSG SozR $2200 \S 551$ Nr. 1; SozR $3-1500 \S 128$ Nr. 11. 
bestandlichen Merkmale der Vollbeweis erforderlich ist, also eine an Gewissheit grenzende Wahrscheinlichkeit. Für die Kausalität gilt aber die Kausalitätstheorie der wesentlichen Bedingung. Und für den Nachweis dieser wesentlichen Bedingung ist hinreichende Wahrscheinlichkeit ausreichend.

Über die im Berufskrankheitenrecht ganz besonders häufig auftretenden Beweisschwierigkeiten ist oft genug Klage geführt worden 38 . Das Schrifttum stand Änderungen ablehnend gegenüber ${ }^{39}$. Auch der Gesetzgeber ist den Reformvorschlägen nicht gefolgt ${ }^{40}$. Herausgekommen ist lediglich die Bestimmung des $\S 9$ Abs. 3 SGB VII. Über die Reichweite dieser Vorschrift gehen die Meinungen allerdings auseinander ${ }^{41}$. Das BSG hatte noch keine Gelegenheit, Position zu dieser Vorschrift zu beziehen.

\section{Die so genannte Quasi-Berufskrankheit (§ 9 Abs. 2 SGB VII)}

Abs. 2 sieht eine erweiterte Entschädigung wegen einer Berufskrankheit vor, auch wenn die Krankheit noch nicht in die Rechtsverordnung aufgenommen ist. Die Vorschrift stellt deshalb einen Kompromiss zwischen einem geschlossenen Listensystem und einer offenen Generalklausel dar ${ }^{42}$. Auch für das Verständnis dieser Vorschrift empfiehlt es sich wieder, den generellen Systemhintergrund der Unfallversicherung stets im Auge zu behalten. Eine Risikozuordnung zur Unfallversicherung (und damit letztlich zu Lasten der Unternehmen) kann nur zulässig sein, wenn es sich um eine Erkrankung aus dem Risikobereich des Unternehmens handelt. Dem trägt der Wortlaut des Abs. 2 auch durchaus Rechnung. Die Krankheit, um deren Entschädigung es geht, unterscheidet sich substanziell in keiner Weise von den Kriterien des Abs. 1. Der einzige Unterschied liegt darin, dass die Krankheit noch nicht formal als Berufskrankheit in die VO aufgenommen worden ist. Und Abs. 2 gibt auch die Antwort darauf, warum von dem in Abs. 1 verankerten Listenprinzip abgewichen werden soll. Es sind die „neuen Erkenntnisse", die ein Abweichen von dem formalen Aufnahmeerfordernis des Abs. 1 erlauben. Mit dieser Konzeption ist die Rationalität der Risikozuordnung gewahrt. Vor diesem Hintergrund versteht es sich auch, weshalb das BSG stets die Auffassung vertreten konnte, dass es sich bei Abs. 2 nicht um eine Härteklausel handelt ${ }^{43}$.

Neue Erkenntnisse liegen vor, wenn sie erst nach Erlass der letzten Liste bekannt geworden sind oder sich erst nach diesem Zeitpunkt zur BK-Reife verdichtet haben ${ }^{44}$.

38 Vgl. zum Themenspektrum, Fuchs, Beweisgrundsätze des zivilen Haftungsrechts und ihre Bedeutung für das Berufskrankheitenrecht, SGb 1997, $601 \mathrm{ff}$.

39 Vgl. etwa Breuer/Velten, NZS 1995, 146 ff.; Krasney, BG 1996, 120 ff.

$40 \mathrm{Zu}$ Reformvorschlägen des Bundesrates s. BT-Drucks. 13/2333, S. 4 ff.

41 Vgl. zu den verschiedenen Positionen Brackmann/Becker, SGB VII, § 9, Rn 221.

42 Ein sog. Mischsystem, vgl. BSGE 44, 90 (91).

43 BSGE 44, 90 (93). Das BVerfG hat diese Rspr. des BSG bestätigt, vgl. BVerfG SozR 3-2200 § 551 Nr. 5.

44 Vgl. Brackmann/Becker, SGB VII, § 9 Rn 309 ff. mit Rspr.-Nachweisen. 
Die Erkenntnisse müssen im Zeitpunkt der Entscheidung (nicht des Auftretens der Erkrankung!) vorliegen.

\section{Stichtagsregelung}

Mit dem Ausdruck verbindet man zunächst etwas höchst Technisches. In Wirklichkeit handelt es sich aber um einen der umstrittensten unbewältigtsten Problemkreise der Berufskrankheitenentschädigung ${ }^{45}$. Das Problem lässt sich sehr einfach beschreiben. Die Normierung von Berufskrankheiten ist in aller Regel ein sich in langen Zeiträumen vollziehender Prozess. Von dem ersten Verdachtsmoment bis zu endgültigen Fixierung einer Berufskrankheit in der BKVO können viele Jahre vergehen. Damit stellt sich die grundlegende Frage, wenn die Listenkrankheit in die Rechtsverordnung aufgenommen ist, für welchen Zeitraum soll die Regelung gelten? Ex nunc? Oder rückwirkend? Wenn Letzteres, wie soll der entscheidende in der Vergangenheit liegende Zeitpunkt bestimmt werden?

Und dies ist eben keine rein technische Frage. Für die Betroffenen Arbeitnehmer kann der Zeitpunkt von fundamentaler Bedeutung sein. Wenn sie in der Vorphase der Normierung erkranken, können sie darauf hoffen, sich auf die Regelung berufen zu können? Oder können sie wenigstens darauf bauen, dass sie über § 9 Abs. 2 SGB VII ihre Rechte durchsetzen können? Oder kann die später erfolgende Normierung als Berufskrankheit die Entschädigung nach § 9 Abs. 2 SGB VII außer Kraft setzen?

Dies ist das Spektrum der Probleme, mit dem wir es zu tun haben. Und hierzu gibt es reichlich Rechtsprechung des BSG und des BVerfG, die ich aus Zeitgründen nicht wiedergeben kann ${ }^{46}$. Nur soviel sei gesagt. Das BSG ist der Auffassung, dass auch der völlige Ausschluss einer Rückwirkungsregelung verfassungsrechtlich nicht zu beanstanden sei. Wenn aber eine Rückwirkung ausgeschlossen ist, müsse sie im Hinblick auf den Gleichheitssatz des Art. 3 GG sachlich gerechtfertigt sein 47 . In der Praxis hat sich das Grundprinzip herausgebildet, das man der Vorschrift des $\S 6$ BKVO entnehmen kann, dass als Rückwirkungsdatum der Tag des In-krafttretens der letzten Änderung der BKVO genommen wird. Das BVerfG hat all dies bis dato nicht beanstandet ${ }^{48}$, mit einer einzigen Ausnahme. Wenn ein Versicherter einen Anspruch aus $§ 9$ Abs. 2 SGB VII geltend macht, darf die Berufsgenossenschaft diesen Anspruch nicht deshalb versagen, weil der Verordnungsgeber die in Betracht kommende Berufskrankheit demnächst in

45 Umfassend dazu Breuer, Die Stichtagsregelung im Berufskrankheitenrecht, in: Boeken/Ruland/ Steinmeyer (Hg.), FS für von Maydell, 2002, S. 125 ff.

46 Eingehend dazu Breuer (Fn. 46), S. $10 \mathrm{ff}$.

47 BSG SozR 3-2200 § 551 Nr. 14.

48 BVerfG SozR 3-2200 § 551 Nr. 15. 
die Liste aufnehmen will und ein Rückwirkungszeitraum vorgesehen ist, in den der Anspruchsteller aber mit dem Beginn seiner Erkrankung nicht fälltt ${ }^{49}$.

\section{Reform}

Gegenwärtig ist eine umfassende Reform der Organisation und des Leistungsrechts der Unfallversicherung in Gang ${ }^{50}$. Was den Wegeunfall anbelangt, soll alles beim Alten bleiben. Lediglich bei rechtskräftigem strafgerichtlichem Urteil wegen einer Verkehrsstraftat soll kein Anspruch auf Gesundheitsschadensausgleich bestehen.

Demgegenüber wird der Berufskrankheitentatbestand mit einigen bedeutsamen Änderungen versehen werden. Zunächst sind einige Änderungen zu vermerken, von denen die Gesetzesbegründung sagt, dass sie die Anerkennungsvoraussetzungen für bestimmte Berufskrankheiten präzisierten. Es genügt in Zukunft nicht mehr, nur die verursachenden Einwirkungen zu bezeichnen, sondern auch die Krankheit selbst. Und wenn wissenschaftliche Erkenntnisse mit hinreichender Sicherheit vorliegen, hat die Bezeichnung außerdem Angaben über Art, Dauer und Ausmaß der Einwirkung zu enthalten. Und ist eine Berufskrankheit in der BKVO nur durch die sie verursachenden Einwirkungen bezeichnet, kann sie im Einzelfall in Zukunft nur noch als Berufskrankheit anerkannt werden, wenn im Zeitpunkt der Entscheidung alle Voraussetzungen einer Berufskrankheit erfüllt sind. Das bedeutet, dass in Zukunft neben der generellen Geeignetheit der Einwirkung, die jeweilige Erkrankung zu verursachen, auch der Nachweis der gruppentypischen Risikoerhöhung erbracht werden muss. Nach Auffassung des Gesetzgebers ist diese Änderung notwendig, weil andernfalls das Listenprinzip ausgehöhlt würde.

Die Möglichkeit der Anerkennung einer Quasi-Berufskrankheit nach Abs. 2 wird aufrechterhalten. Allerdings wird in Satz 2 eine Sperrwirkung vorgesehen. Prüft nämlich der Verordnungsgeber die Aufnahme in die Liste, darf eine Entscheidung nach Satz 1 nicht getroffen werden. Das BMA muss deshalb jeweils zu Beginn eines Jahres die Krankheiten bekannt geben, bei denen eine Bezeichnung geprüft wird. Die Sperrwirkung endet spätestens drei Jahre nach der erstmaligen Bekanntgabe. Der Gesetzesbegründung kann man entnehmen, dass die Anerkennung von Berufskrankheiten damit strikt nach einem rechtlichen, hierarchisch gegliederten Vorschriftensystem erfolgen soll. Grundsätzlich gilt nach $\S 9$ Abs. 1 und dem neuen Abs. 1 a ein Entscheidungsvorbehalt des Verordnungsgebers. Die Anerkennung nach Abs. 2 hat demgegenüber Ausnahmecharakter. Die Sperrklausel entspricht dem gesetzlich festgelegten Verhältnis

49 BVerfG SozR 4-1100 Art. 3, Nr. 32. Ebenso für eine ähnliche Fallgestaltung BSG NZS 2007, 325 (327).

50 Eckpunkte der Bund-Länder-Arbeitsgruppe vom 29.6.2006 sowie Arbeitsentwurf eines Gesetzes zur Reform der gesetzlichen Unfallversicherung vom 27. April 2007 zur Reform der gesetzlichen Unfallversicherung. 
zwischen dem Enumerationsprinzip der Berufskrankheitenliste und der Ausnahmeklausel des Abs. 2. Greift der Verordnungsgeber eine entsprechende Fragestellung auf, würde eine unbeschränkt fortbestehende Entscheidungsfreiheit von Unfallversicherungsträgern und Gerichten einen weiten Eingriff in die Regelungskompetenz des Verordnungsgebers darstellen.

Die in Aussicht genommene neue Regelung bringt schließlich auch eine Lösung des Stichtagsproblems. Ein Versicherungsfall ist in Zukunft ausgeschlossen, wenn die Krankheit mehr als zehn Jahre vor dem Zeitpunkt eingetreten ist, in dem sie einem Unfallversicherungsträger bekannt geworden ist. Von diesem Zeitpunkt an werden auch Renten wegen Berufskrankheiten geleistet. Der Gesetzgeber beabsichtigte damit eine einheitliche Regelung unabhängig von der anzuwendenden Anspruchsgrundlage. 
